\title{
Thickness measurement of metallic film based on a high-frequency feature of triple-coil electromagnetic eddy current sensor
}

\author{
Mingyang Lu*, Liming Chen, Xiaobai Meng, Ruochen Huang*, Anthony Peyton, Wuliang Yin*
}

\begin{abstract}
Previously, various techniques have been proposed for reducing the lift-off effect on the thickness measurement of the non-magnetic films, including the peak-frequency feature and phase feature in the Dodd-Deed analytical formulation. To realise a real-time feedback response on the thickness monitoring, the phase term in the Dodd-Deeds formulation must be taken off the integration. Previous methods were based on the slow change rate of the phase term when compared to the rest of the term - the magnitude term. However, the change rate of the phase term is still considerable for a range of working frequencies. In this paper, a high-frequency feature has been found. That is, the ratio between the imaginary and real part of the phase term is proportional to the integral variable $\alpha$ under high frequencies. Based on this proportion relationship, the phase term has been taken out; and a thickness algorithm has been proposed. By combing the measured impedance from the custom-built sensor (three coils), the thickness of the metallic film can be reconstructed. Experiments have been carried out for the verification of the proposed scenario. Results show that the thickness of the metal film can be reconstructed with a small error of less than $2 \%$, and immune to a reasonable range of lift-offs.
\end{abstract}

Index Terms - Eddy current testing, thickness measurement, non-destructive testing, lift-off, real-time monitoring.

\section{INTRODUCTION}

$\mathrm{E}$ LECTROMAGNETIC eddy current testing (ECT) has been commonly used for diverse applications of the sample checking, including measuring the properties inhomogeneity, surface inspection in order to further improve the product quality [1-20]. Besides the ECT method, other non-destructive testing techniques like ultrasonic have been wildly used [21], which is more preciously on the surface inspection of metals. However, ultrasonic sensing is difficult on the measurement of thin materials. As one application of ECT, thickness measurement of the metallic film can be achieved by referring to the measurement signal - mutual impedance or inductance of the sensing coils. However, the lift-off of the sensor could significantly influence the measured result.

Approaches of using both the pulsed eddy current (PEC) and multi-frequency have been proposed on reducing the liftoff effects when measuring the thickness of the metallic film [22-24]. For the PEC method, by injecting a time-domain

This work was supported by [UK Engineering and Physical Sciences Research Council (EPSRC)] [grant number: EP/P027237/1] [title: Real-time Inline Microstructural Engineering (RIME)].

M. Lu (Member, IEEE), R. Huang, L. Chen, A. Peyton and W. Yin (Senior Member, IEEE) are with the School of Electrical and Electronic Engineering, University of Manchester, Sackville Street Building, Manchester, M13 9PL, differential-pulsed excitation current, the characteristic of the sample (including the thickness) can be derived from the signal feature. For example, a two-reference signal method has been used to reduce the lift-off effect [25]. Moreover, a look-up table has been introduced by Tai et al, which is extracted from a custom designed instrument on the thickness measurement [26]. For the multi-frequency eddy current method, by using a digital processing technique based on Walsh functions, Pinotti et al. proposed a novel property measurement method of the metallic alloy [27].

Previously, various techniques have been proposed to reduce the lift-off effect on the thickness of the metallic, including the peak frequency feature, phase feature of the impedance or inductance [28-31]. Most of these methods are based on the slow changing rate of the phase term $\phi(\alpha)$, which was taken out of the integration in the Dodd-Deeds model for further fastening the reconstruction (especially for the real-time monitoring application). Although the thickness measurement from the previous proposed algorithm is almost immune to the lift-off distance, the change rate of the phase term is still considerable under a range of frequencies or sample properties. Consequently, the spatial frequency (which was believed merely determined by the sensor) needed to be re-fitted and updated for different working frequencies.

In this paper, to address the lift-off issue on the thickness measurement of metallic films, a novel algorithm has been proposed based on a high-frequency feature from the designed sensor feedback. That is the ratio between the imaginary part and real part of the phase term $-\frac{\operatorname{Im}(\phi(\alpha))}{\operatorname{Re}(\phi(\alpha))}$ is found to be proportional to $\alpha$ in the Dodd-Deeds formulation. With this relationship, the spatial frequency is a 'true' constant with respect to different frequencies. The sensor is composed of three circular coils, with one transmitter coil and two-receiver coils enwound of different radii. The thickness of the nonmagnetic metal film can be reconstructed via the proposed algorithm and combined signal (measured electrical resistance) of two sensing pairs. Experiments have been carried out for the performance test and verification of the proposed thickness measurement scenario.

UK (mingyang.lu@manchester.ac.uk; ruochen.huang@manchester.ac.uk; wuliang.yin@manchester.ac.uk)

*Corresponding author: M. Lu, R. Huang, W. Yin.

$\mathrm{X}$. Meng is with the Faculty of Art, Science and Technology, University of Northampton, Northampton, NN1 5PH, UK 


\section{FORMULATION ON THE THICKNESS MEASUREMENT OF METAL PLATE}

From our previous work, it has been found the lift-off of the sensor could significantly affect the sensor response including the induced voltage, impedance, or mutual inductance of the transmitter and receiver $[32,33]$. The accuracy of the sensor response is vital for the reconstruction of the sample properties. Since different dimensions of the sensor could get different sensor responses, a hypothesis of combining two sensing pairs with different coil dimensions could be a solution for reducing the lift-off effect. Thus, as shown in Fig. 1, an air-core sensor is designed, with one transmitter and two receiver coils enwound on the same lift-off plane.

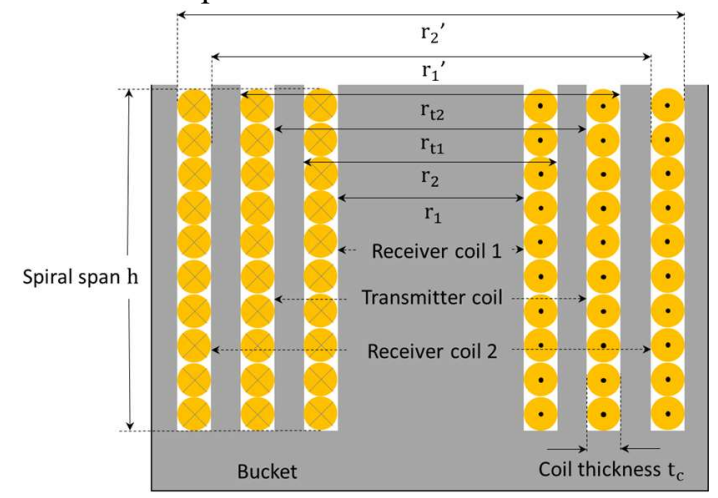

Fig. 1 Air-core sensor setup - three coils co-axially enwound on a same lift-off

Assume the sensing coils in all the coils have the same turns $\mathrm{N}$, spiral span $\mathrm{h}$ and coil thickness $\mathrm{t}_{\mathrm{c}}$, then the impedance change (subtract of the impedance for the presence and absence of the specimen) tested by two sensing pairs can be derived from the Dodd-Deeds formulation [34],

$$
\begin{aligned}
& \Delta \mathrm{Z}_{1}(\omega)=\mathrm{j} \omega \overline{\mathrm{r}_{1}} \mathrm{~K} \int_{0}^{\infty} \frac{\mathrm{P}_{1}(\alpha)}{\alpha^{6}} \mathrm{~A}(\alpha) \phi(\alpha) \mathrm{d} \alpha \\
& \Delta \mathrm{Z}_{2}(\omega)=\mathrm{j} \omega \overline{\mathrm{r}_{2}} \mathrm{~K} \int_{0}^{\infty} \frac{\mathrm{P}_{2}(\alpha)}{\alpha^{6}} \mathrm{~A}(\alpha) \phi(\alpha) \mathrm{d} \alpha
\end{aligned}
$$

In equation (1),

$$
\mathrm{K}=\frac{\pi \mathrm{N}^{2} \mu_{0}}{\mathrm{~h}^{2} \mathrm{t}_{\mathrm{c}}{ }^{2}}
$$

$\overline{r_{1}}$, and $\overline{r_{2}}$ are the mean radii of (the outer and inner radii) the receiver 1 and 2 .

$$
\begin{gathered}
\overline{\mathrm{r}_{1}}=\frac{\mathrm{r}_{1}+\mathrm{r}_{2}}{2} \\
\overline{\mathrm{r}_{2}}=\frac{\mathrm{r}_{1}{ }^{\prime}+\mathrm{r}_{2}{ }^{\prime}}{2} \\
\phi(\alpha)=\frac{\left(\alpha_{1}+\mu_{1} \alpha\right)\left(\alpha_{1}-\mu_{1} \alpha\right)-\left(\alpha_{1}+\mu_{1} \alpha\right)\left(\alpha_{1}-\mu_{1} \alpha\right) \mathrm{e}^{2 \alpha_{1} \mathrm{c}}}{-\left(\alpha_{1}-\mu_{1} \alpha\right)\left(\alpha_{1}-\mu_{1} \alpha\right)+\left(\alpha_{1}+\mu_{1} \alpha\right)\left(\alpha_{1}+\mu_{1} \alpha\right) \mathrm{e}^{2 \alpha_{1} \mathrm{c}}} \\
\alpha_{1}=\sqrt{\alpha^{2}+j \omega \sigma \mu_{1} \mu_{0}} \\
\mathrm{~A}(\alpha)=\mathrm{e}^{-2 \alpha \mathrm{l}_{0}}\left(\mathrm{e}^{-2 \alpha \mathrm{h}}-2 \mathrm{e}^{-\alpha \mathrm{h}}+1\right) \\
\mathrm{P}_{1}(\alpha)=\int_{\alpha \mathrm{r}_{\mathrm{t} 1}}^{\alpha \mathrm{r}_{\mathrm{t} 2}} \tau \mathrm{J}_{1}(\tau) \mathrm{d} \tau \int_{\alpha \mathrm{r}_{1}}^{\alpha \mathrm{r}_{2}} \tau \mathrm{J}_{1}(\tau) \mathrm{d} \tau \\
\mathrm{P}_{2}(\alpha)=\int_{\alpha \mathrm{r}_{\mathrm{t} 1}}^{\alpha \mathrm{r}_{\mathrm{t} 2}} \tau \mathrm{J}_{1}(\tau) \mathrm{d} \tau \int_{\alpha \mathrm{r}_{1^{\prime}}}^{\alpha \mathrm{r}_{2^{\prime}}} \tau \mathrm{J}_{1}(\tau) \mathrm{d} \tau
\end{gathered}
$$

Where, $r_{t 1}, r_{1}$, and $r_{1}{ }^{\prime}$ are the inner radii of the transmitter, receiver 1 , and receiver $2 . r_{t 2}, r_{2}$, and $r_{2}{ }^{\prime}$ are the outer radii of the transmitter, receiver 1 , and receiver $2 . \omega$ is the working angular frequency. $\mathrm{N}$ denotes the turns of the transmitter and receiver spiral coils. $\mu_{0}$ is the vacuum permeability. $\mu_{1}$ depicts the relative permeability of the specimen. $\mathrm{c}$ is the thickness of the metallic plate. $\sigma$ is the electrical conductivity of the specimen. $\mathrm{l}_{0}$ is the lift-off of the sensor. $\mathrm{J}_{1}$ is the first order Bessel function of the first kind. $\tau$ and $\alpha$ denote the integrated parameters.

Through further deductions from equation (1), the impedance change of transmitter-receiver 1 can be divided as its real and imaginary integration parts.

$$
\begin{aligned}
& \operatorname{Re}\left(\Delta \mathrm{Z}_{1}(\omega)\right)=-\omega \overline{\mathrm{r}_{1}} \mathrm{~K} \int_{0}^{\infty} \frac{\mathrm{P}_{1}(\alpha)}{\alpha^{6}} \mathrm{e}^{-2 \alpha \mathrm{l}_{0}}\left(\mathrm{e}^{-2 \alpha \mathrm{h}}-2 \mathrm{e}^{-\alpha \mathrm{h}}+1\right) \operatorname{Im}(\phi(\alpha)) \mathrm{d} \alpha \\
& \operatorname{Im}\left(\Delta \mathrm{Z}_{1}(\omega)\right)=\omega \overline{\mathrm{r}_{1}} \mathrm{~K} \int_{0}^{\infty} \frac{\mathrm{P}_{1}(\alpha)}{\alpha^{6}} \mathrm{e}^{-2 \alpha \mathrm{l}_{0}}\left(\mathrm{e}^{-2 \alpha \mathrm{h}}-2 \mathrm{e}^{-\alpha \mathrm{h}}+1\right) \operatorname{Re}(\phi(\alpha)) \mathrm{d} \alpha
\end{aligned}
$$

Previously, the phase term $\phi(\alpha)$ was taken out of the integration considering it changes slowly with respect to $\alpha$ when compared with the magnitude part. Consequently, the multi-frequency features including the peak frequency for the non-magnetic materials and zero-crossing frequency for the magnetic materials have been formulated [29, 33]. However, it has been found the change rate of the phase term $\phi(\alpha)$ cannot be neglected under a range of working frequencies.

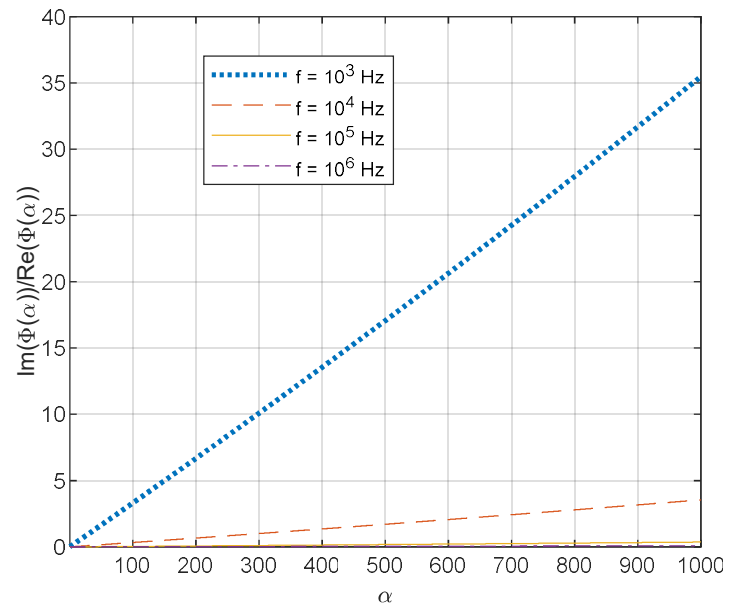

(a)

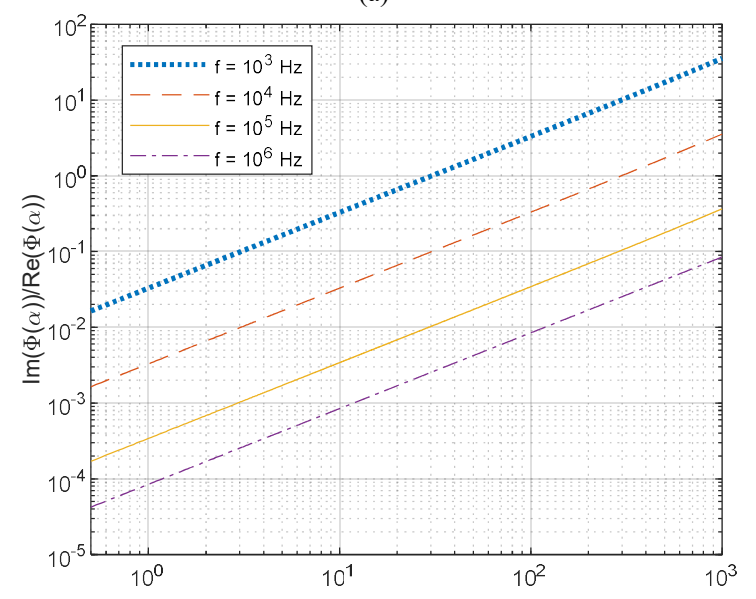

(b)

Fig. $2 \frac{\operatorname{Im}(\phi(\alpha))}{\operatorname{Re}(\phi(\alpha))}$ is linear with the variable $\alpha$ for different frequencies a) linear plot b) log-log plot

As Fig. 2 depicts, for the non-magnetic material, $\mu_{1}=1$. The term $\frac{\operatorname{Im}(\phi(\alpha))}{\operatorname{Re}(\phi(\alpha))}$ is found to be linear with the variable $\alpha$. The 
proportional factor is defined as $\mathrm{T}(\omega)$.

$$
\operatorname{Im}(\phi(\alpha))=\mathrm{T}(\omega) \alpha \operatorname{Re}(\phi(\alpha))
$$

For a certain sample and working frequency, the proportional factor $T(\omega)$ is a constant.

The solution of $\mathrm{T}$ can be derived as,

$$
\mathrm{T}(\omega)=\lim _{\alpha \rightarrow 0} \frac{\operatorname{Im}(\phi(\alpha))}{\alpha \operatorname{Re}(\phi(\alpha))}
$$

Through mathematical manipulations using Matlab symbolic variables and functions, equation (14) becomes,

$$
\mathrm{T}(\omega)=\operatorname{Im}\left(\frac{(\mathrm{j}-1) \sqrt{2}\left(\mathrm{e}^{(1+\mathrm{j}) \mathrm{c} \sqrt{2 \omega \sigma \mu_{0}}}+1\right)}{\sqrt{\omega \sigma \mu_{0}}\left(\mathrm{e}^{(1+\mathrm{j}) \mathrm{c} \sqrt{2 \omega \sigma \mu_{0}}}-1\right)}\right)
$$

Equation (15) can be further deduced as,

$$
\mathrm{T}(\omega)=\frac{\sqrt{2}\left(1+\mathrm{e}^{2 c \sqrt{2 \omega \sigma \mu_{0}}}\right)}{\sqrt{\omega \sigma \mu_{0}}\left(\mathrm{e}^{2 c \sqrt{2 \omega \sigma \mu_{0}}}-2 \mathrm{e}^{\mathrm{c} \sqrt{2 \omega \sigma \mu_{0}}} \cos \left(\mathrm{c} \sqrt{2 \omega \sigma \mu_{0}}\right)+1\right)}
$$

For the thin-film metallic plate, (16) can be simplified through the Padé approximation.

$$
\mathrm{T}(\omega)=\frac{1}{\sqrt{2 \omega \sigma \mu_{0}}}\left(1+\frac{1}{\mathrm{c}^{2} \omega \sigma \mu_{0}}\right)
$$

As can be observed from Fig. 3 (a) that the real part of $\operatorname{Re}(\phi(\alpha))$ term varies slowly with $\alpha$ under higher frequencies. A small value function $G$ is used to approximate $\operatorname{Re}(\phi(\alpha))$. From (2), since $\phi(\alpha)$ is determined by the sample and working frequency instead of the sensor parameters, the function $G$ is merely controlled by the variable $\omega, \sigma$, and c. Therefore, for a high working frequency,

$$
\operatorname{Re}(\phi(\alpha))=-\mathrm{e}^{-2 \alpha G(\omega, \sigma, c)}
$$

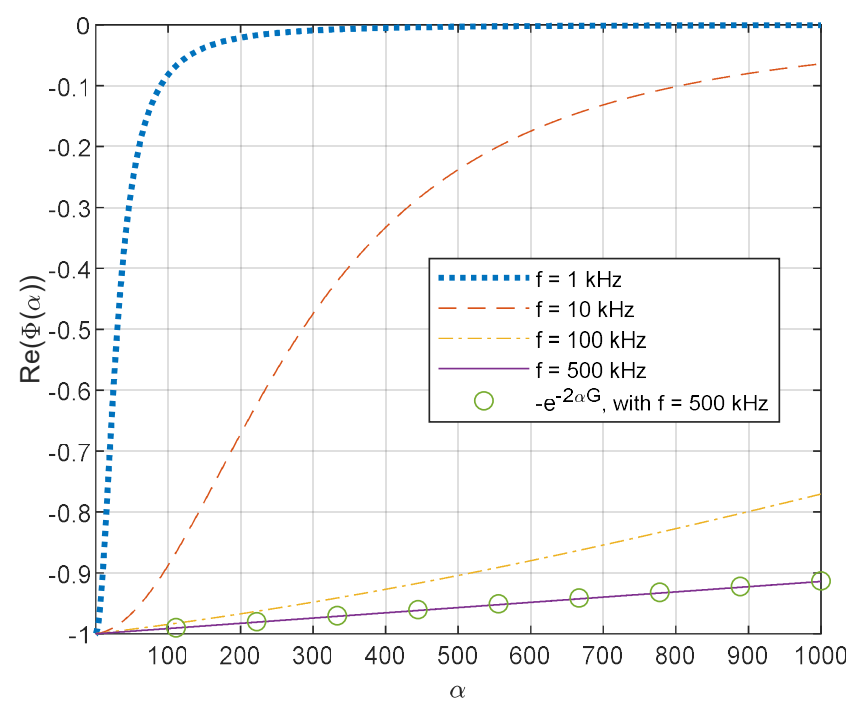

Fig. $3 \operatorname{Re}(\phi(\alpha))$ versus $\alpha$ for different frequencies $\left(-e^{-2 \alpha G(\omega, \sigma, c)}\right.$ is used to estimate $\operatorname{Re}(\phi(\alpha))$ under $500 \mathrm{kHz})$

Substitute (13) (18) into (11) and (12), the real and imaginary parts of the impedance change from transmitter-receiver 1 under high working frequencies are,

$$
\begin{aligned}
& \operatorname{Re}\left(\Delta \mathrm{Z}_{1}(\omega)\right) \\
& =\omega \mathrm{T}(\omega) \overline{r_{1}} \mathrm{~K} \int_{0}^{\infty} \frac{\mathrm{P}_{1}(\alpha)}{\alpha^{5}} \mathrm{e}^{-2 \alpha \mathrm{l}_{0}}\left(\mathrm{e}^{-2 \alpha \mathrm{h}}-2 \mathrm{e}^{-\alpha \mathrm{h}}+1\right) \mathrm{e}^{-2 \alpha G(\omega, \sigma, \mathrm{c})} \mathrm{d} \alpha \\
& \operatorname{Im}\left(\Delta \mathrm{Z}_{1}(\omega)\right) \\
& \quad=-\omega \overline{\mathrm{r}_{1}} \mathrm{~K} \int_{0}^{\infty} \frac{\mathrm{P}_{1}(\alpha)}{\alpha^{6}} \mathrm{e}^{-2 \alpha \mathrm{l}_{0}}\left(\mathrm{e}^{-2 \alpha \mathrm{h}}-2 \mathrm{e}^{-\alpha \mathrm{h}}+1\right) \mathrm{e}^{-2 \alpha \mathrm{G}(\omega, \sigma, c)} \mathrm{d} \alpha
\end{aligned}
$$

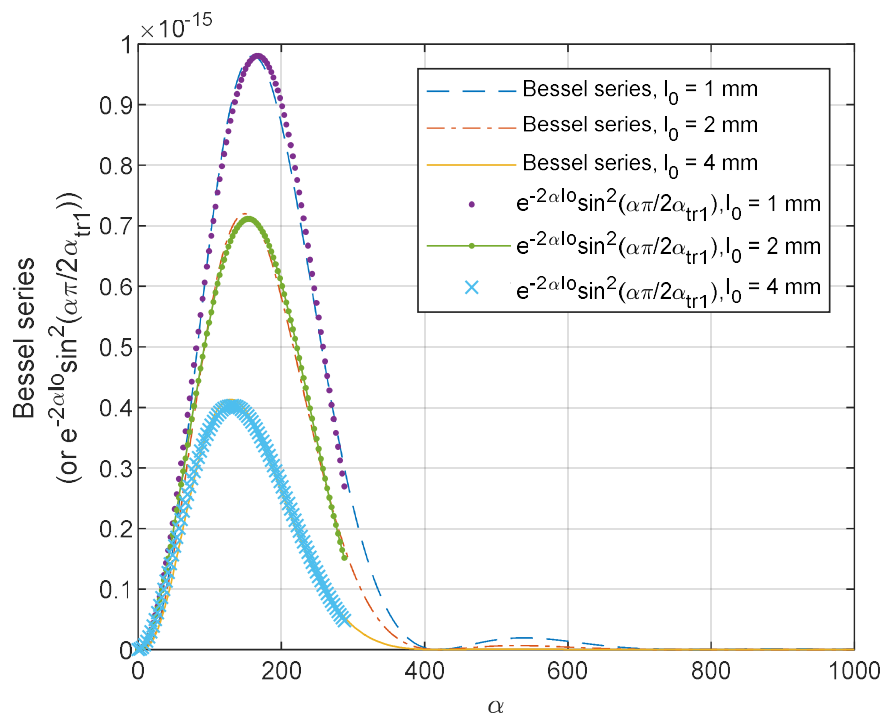

Fig. 4 Approximation of the Bessel series

As can be seen from Fig. 4, the Bessel series $\frac{P_{1}(\alpha)}{\alpha^{5}} e^{-2 \alpha l_{0}}\left(e^{-2 \alpha h}-\right.$ $\left.2 \mathrm{e}^{-\alpha \mathrm{h}}+1\right)$ can be estimated as a sinusoidal function multiplied by the lift-off decay factor $\mathrm{e}^{-2 \alpha \mathrm{l}_{0}}$. Therefore, the integration term in (19) becomes,

$$
\begin{aligned}
& \int_{0}^{\infty} \frac{\mathrm{P}_{1}(\alpha)}{\alpha^{5}} \mathrm{e}^{-2 \alpha\left(\mathrm{l}_{0}+\mathrm{G}\right)}\left(\mathrm{e}^{-2 \alpha \mathrm{h}}-2 \mathrm{e}^{-\alpha \mathrm{h}}+1\right) \mathrm{d} \alpha \\
& =\Delta \mathrm{Z}_{m 1} \int_{0}^{2 \alpha_{\mathrm{rr} 1}} \mathrm{e}^{-2\left(\mathrm{l}_{0}+\mathrm{G}\right)} \sin ^{2}\left(\frac{\alpha \pi}{2 \alpha_{\mathrm{tr1}}}\right) \mathrm{d} \alpha \\
& =\Delta \mathrm{Z}_{m 1} \frac{\pi^{2}\left(1-\mathrm{e}^{-4 \alpha_{\mathrm{tr} 1}\left(\mathrm{l}_{0}+\mathrm{G}\right)}\right)}{4\left(\mathrm{l}_{0}+\mathrm{G}\right)\left(4 \alpha_{\mathrm{tr} 1}{ }^{2}\left(\mathrm{l}_{0}+\mathrm{G}\right)^{2}+\pi^{2}\right)}
\end{aligned}
$$

$\alpha_{\text {tr } 1}$ is a constant and named as the spatial frequency for the transmitter and receiver 1 sensing pair. $\Delta \mathrm{Z}_{m 1}$ denotes the normalization factor, which determines the ratio between the Bessel series and sinusoidal function and can be defined at the peak point when $\alpha=\alpha_{\mathrm{tr} 1}$,

$$
\Delta \mathrm{Z}_{m 1}=\frac{\mathrm{P}_{1}\left(\alpha_{\mathrm{tr} 1}\right)}{\alpha_{\mathrm{tr} 1}{ }^{5}}\left(\mathrm{e}^{-2 \alpha_{\mathrm{tr} 1} \mathrm{~h}}-2 \mathrm{e}^{-\alpha_{\mathrm{tr} 1} \mathrm{~h}}+1\right)
$$

With a small sensor lift-off $l_{0}$, according to the Padé approximation, $\mathrm{e}^{-4 \alpha_{\operatorname{tr} 1}\left(\mathrm{l}_{0}+\mathrm{G}\right)}$ can be estimated as (1$\left.4 \alpha_{\mathrm{tr} 1}\left(\mathrm{l}_{0}+\mathrm{G}\right)\right)$. Therefore, (21) becomes,

$$
\begin{aligned}
& \int_{0}^{\infty} \frac{\mathrm{P}_{1}(\alpha)}{\alpha^{5}} \mathrm{e}^{-2 \alpha\left(\mathrm{l}_{0}+\mathrm{G}\right)}\left(\mathrm{e}^{-2 \alpha \mathrm{h}}-2 \mathrm{e}^{-\alpha \mathrm{h}}+1\right) \mathrm{d} \alpha \\
& =\Delta \mathrm{Z}_{m 1} \frac{\alpha_{\mathrm{tr} 1} \pi^{2}}{\left(4 \alpha_{\mathrm{tr} 1}{ }^{2}\left(\mathrm{l}_{0}+\mathrm{G}\right)^{2}+\pi^{2}\right)}
\end{aligned}
$$

Substitute (23) into (19), the real part of the impedance from transmitter-receiver 1 sensing pair becomes,

$$
\operatorname{Re}\left(\Delta \mathrm{Z}_{1}(\omega)\right)=\Delta \mathrm{Z}_{m 1} \frac{\alpha_{\mathrm{tr} 1} \pi^{2} \omega \mathrm{T}(\omega) \overline{\mathrm{r}_{1}} \mathrm{~K}}{\left(4 \alpha_{\mathrm{tr} 1}{ }^{2}\left(\mathrm{l}_{0}+\mathrm{G}\right)^{2}+\pi^{2}\right)}
$$

It can be seen from (24) that $\operatorname{Re}\left(\Delta \mathrm{Z}_{1}(\omega)\right)$ can be calculated by a simple equation instead of integrating over the entire $\alpha$ domain.

Similarly, the real part of the impedance from transmitterreceiver 2 sensing pair is,

$$
\operatorname{Re}\left(\Delta \mathrm{Z}_{2}(\omega)\right)=\Delta \mathrm{Z}_{m 2} \frac{\alpha_{\mathrm{tr} 2} \pi^{2} \omega \mathrm{T}(\omega) \overline{\mathrm{r}_{2}} \mathrm{~K}}{\left(4 \alpha_{\mathrm{tr2} 2}{ }^{2}\left(\mathrm{l}_{0}+\mathrm{G}\right)^{2}+\pi^{2}\right)}
$$

In equation (25), $\alpha_{\operatorname{tr} 2}$ is the spatial frequency for the transmitter and receiver 2 sensing pair. And, 


$$
\Delta \mathrm{Z}_{m 2}=\frac{\mathrm{P}_{2}\left(\alpha_{\mathrm{tr} 2}\right)}{\alpha_{\mathrm{tr} 2} 5}\left(\mathrm{e}^{-2 \alpha_{\mathrm{tr} 2} \mathrm{~h}}-2 \mathrm{e}^{-\alpha_{\mathrm{tr} 2} \mathrm{~h}}+1\right)
$$

Assume the real part of the impedance are $\mathrm{R}_{1}$, andR $\mathrm{R}_{2}$. I.e. $\mathrm{R}_{1}=\operatorname{Re}\left(\Delta \mathrm{Z}_{1}(\omega)\right), \mathrm{R}_{2}=\operatorname{Re}\left(\Delta \mathrm{Z}_{2}(\omega)\right)$, combine (24) with (25),

$$
\mathrm{T}(\omega)=\frac{\mathrm{R}_{1}\left(4 \alpha_{\mathrm{tr} 1}{ }^{2}\left(\mathrm{l}_{0}+\mathrm{G}\right)^{2}+\pi^{2}\right)}{\alpha_{\mathrm{tr} 1} \pi^{2} \omega \overline{\mathrm{r}_{1}} \mathrm{~K} \Delta \mathrm{Z}_{m 1}}=\frac{\mathrm{R}_{2}\left(4 \alpha_{\mathrm{tr2}}{ }^{2}\left(\mathrm{l}_{0}+\mathrm{G}\right)^{2}+\pi^{2}\right)}{\alpha_{\mathrm{tr} 2} \pi^{2} \omega \overline{\mathrm{r}_{2}} \mathrm{~K} \Delta \mathrm{Z}_{m 2}}
$$

Further deductions from (27), the lift-off term can be substituted as,

$$
\left(l_{0}+G\right)^{2}=\frac{\pi^{2}\left(\alpha_{t r 1} \overline{r_{1}} \Delta Z_{m 1} R_{2}-\alpha_{t r 2} \overline{r_{2}} \Delta Z_{m 2} R_{1}\right)}{4 \alpha_{t r 1} \alpha_{t r 2}\left(\alpha_{t r 1} \overline{r_{2}} \Delta Z_{m 2} R_{1}-\alpha_{t r 2} \overline{r_{1}} \Delta Z_{m 1} R_{2}\right)}
$$

Then substitute $\left(\mathrm{l}_{0}+\mathrm{G}\right)^{2}$ into $(27), \mathrm{T}(\omega)$ can be derived.

$$
\mathrm{T}(\omega)=\frac{\left(\alpha_{\mathrm{tr} 1}{ }^{2}-\alpha_{\mathrm{tr2}}{ }^{2}\right) \mathrm{R}_{1} \mathrm{R}_{2}}{\left(\alpha_{\mathrm{tr} 1} \overline{\mathrm{r}_{2}} \Delta \mathrm{Z}_{m 2} \mathrm{R}_{1}-\alpha_{\mathrm{tr} 2} \overline{\mathrm{r}_{1}} \Delta \mathrm{Z}_{m 1} \mathrm{R}_{2}\right) \mathrm{K} \alpha_{\mathrm{tr} 1} \alpha_{\mathrm{tr} 2} \omega}
$$

Combine (29) with (17) under the single frequency $\omega$, the thickness of the thin film plate can be reconstructed as,

$$
c=\frac{1}{\sqrt{\frac{\left(\alpha_{t r 1}^{2}-\alpha_{t r 2}^{2}\right) R_{1} R_{2} \sigma \mu_{0} \sqrt{2 \omega \sigma \mu_{0}}}{\left(\alpha_{t r 1} \overline{\mathrm{r}}_{2} \Delta \mathrm{Z}_{m 2} \mathrm{R}_{1}-\alpha_{\mathrm{tr} 2} \overline{\mathrm{r}}_{1} \Delta \mathrm{Z}_{m 1} \mathrm{R}_{2}\right) \mathrm{K} \alpha_{\mathrm{tr} 1} \alpha_{\mathrm{tr} 2}}-\omega \sigma \mu_{0}}}
$$

Where, $\Delta \mathrm{Z}_{m 1}$ and $\Delta \mathrm{Z}_{m 2}$ are defined in (22) and (26).

From equation (30), the thickness of the non-magnetic thin film can be reconstructed from the measured resistance (real part of the impedance) of transmitter-receiver 1 and transmitterreceiver 2 sensing pairs. It can be observed from (30) that, the reconstructed thickness could be more accurate under a bigger difference between either dimensions of sensing pair (related to $\alpha_{\operatorname{tr} 1}$ and $\left.\alpha_{\operatorname{tr} 2}\right)$, and or the measured resistance $\left(R_{1}-R_{2}\right.$, which generally increases with frequencies), and large working frequencies.

\section{EXPERIMENTAL VERIFICATION}

To verify the feasibility of the designed sensor and the performance of the thickness formulation in equation (30). Experiments have been carried out for the mutual impedance measurement using the designed sensor under different frequencies and lift-offs.

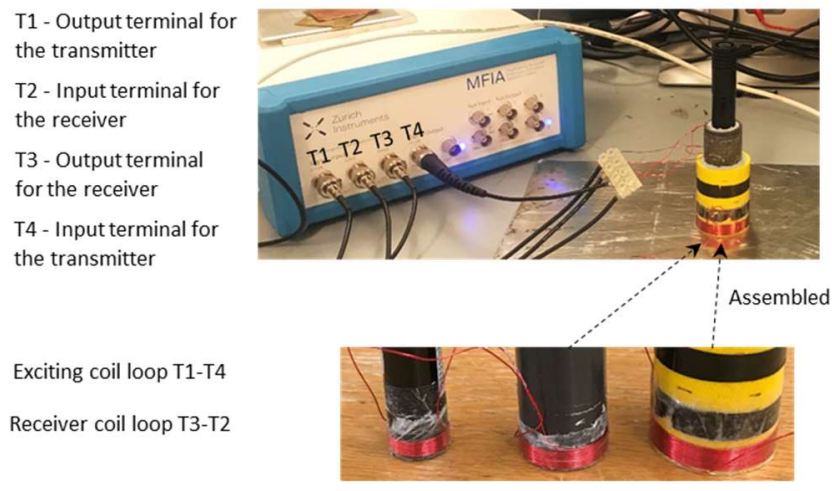

(a)

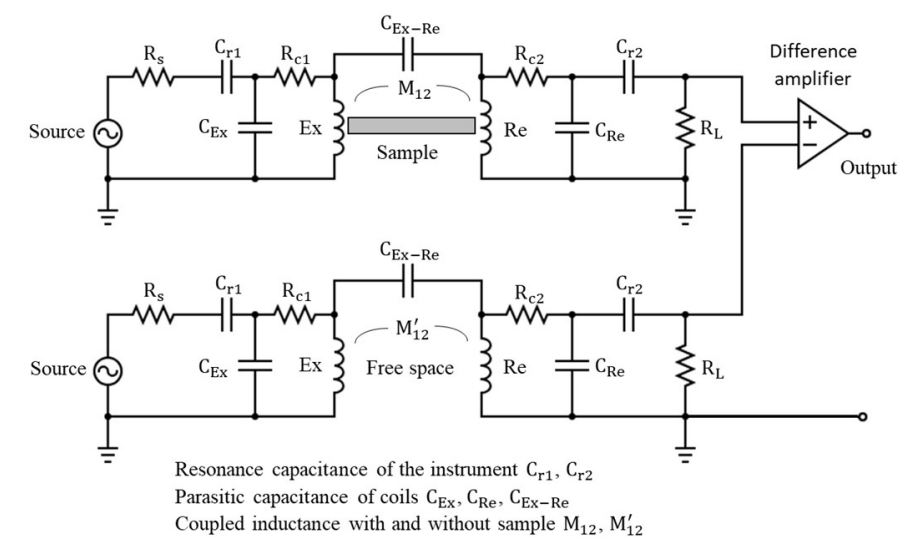

(b)

Fig. 5 (a) Experiment setup for the measurement of the electromagnetic impedance (b) Equivalent circuit of the measurement setup

As Fig. 5 depicts, the copper wire with a thickness of 0.25 $\mathrm{mm}$ is seamlessly enwound on three plastic cylinder rods. As demonstrated in Table 1, 3 coils have the same number of turns, spiral span, and lift-offs. The lift-off was controlled by the plastic lift-off spacers and ranges from $1 \mathrm{~mm}$ to $6 \mathrm{~mm}$. The sensor was deployed on the films and its coils were connected to the Zurich impedance analyser for the multi-frequency impedance measurement. The operation frequency of the Zurich impedance analyser ranges from $1 \mathrm{kHz}$ to $500 \mathrm{kHz}$. Lower frequencies will result in a reduced signal-to-noise ratio (SNR). In addition, the maximum of the frequency was capped at $500 \mathrm{kHz}$ to ensure that no skin or diffusion effects exist (so that the electromagnetic field could penetrate deeply enough to interact or reflect with respect to the bottom surface of the metallic film). The skin depth as a function of frequency is shown in (31). For the measurement, the parasitic capacitance is an unavoidable and unwanted capacitance that exists between the sensor plates, and adjacent coils due to their proximity. As illustrated in Fig. 5, to address the measurement issue due to the proximity effect, the reference signals have been measured. By subtracting the reference signals when the sensor is deployed in the free space, the ambient noise signals can be significantly eliminated. Besides, since the maximum frequency is only 500 $\mathrm{kHz}$ (far away from the resonance frequency), the parasitic capacitance of the coil is not an issue.

$$
\rho=\frac{1}{\sqrt{\pi f \sigma \mu_{1} \mu_{0}}}
$$

TABLE I

SENSOR PARAMETERS

\begin{tabular}{cccc}
\hline \hline & Receiver 1 & Transmitter & Receiver 2 \\
\hline Inner radius (mm) & 9.00 & 13.50 & 19.50 \\
Outer radius (mm) & 9.25 & 13.75 & 19.75 \\
Turns & & 20 & \\
Coils spiral span (mm) & \multicolumn{3}{c}{5.00} \\
Lift-offs (mm) & \multicolumn{3}{c}{$1.00: 1.00: 6.00$} \\
\hline \hline
\end{tabular}

For the non-magnetic materials, as illustrated in Table 2, the aluminium and copper films with the electrical conductivity of $35 \mathrm{MS} / \mathrm{m}, 57 \mathrm{MS} / \mathrm{m}$, and thickness of $44 \mu \mathrm{m}$ (or $66 \mu \mathrm{m}$ ), $30 \mu \mathrm{m}$ (or $60 \mu \mathrm{m}$ ) have been utilised for the experiment. The electrical conductivity of the aluminium and copper films has been 
measured in previous research via the four-terminal sensing method.

TABLE II

PARAMETERS OF THE METALLIC FILMS

\begin{tabular}{ccc}
\hline \hline & Aluminium & Copper \\
\hline Actual thickness $(\mu \mathrm{m})$ & 44,66 & 30,60 \\
$\begin{array}{c}\text { Electrical conductivity } \\
(\mathrm{MS} / \mathrm{m})\end{array}$ & 35 & 57 \\
\hline \hline
\end{tabular}

\section{RESULTS}

\section{A. Measurement of sensor response}

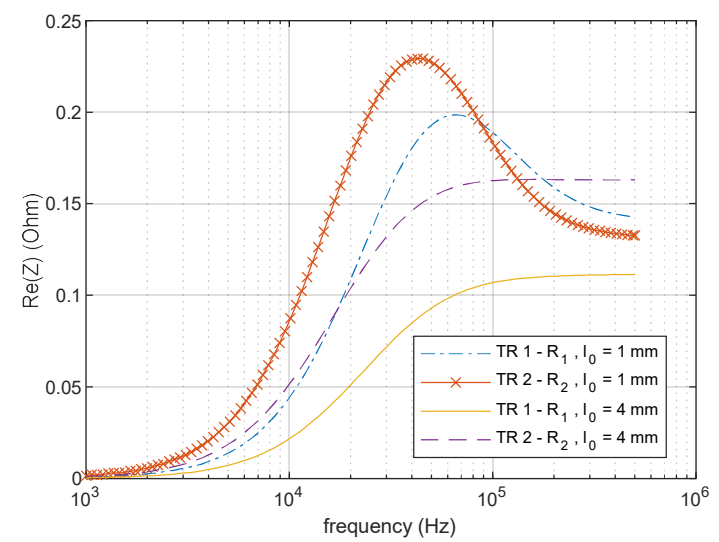

(a)

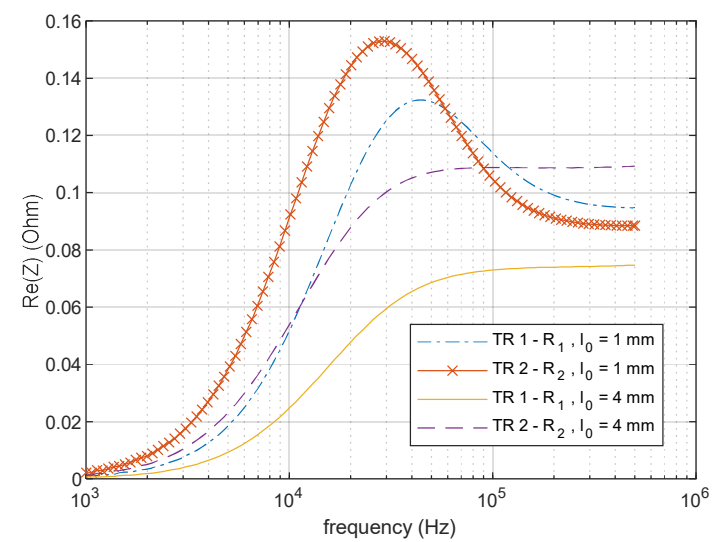

(b)

Fig. 6 Experimental data for the multi-frequency electrical resistance (real part of the measured impedance) of aluminium films under the sensor lift-off of 1 and $4 \mathrm{~mm}$ (a) $44 \mu \mathrm{m}$ (b) $66 \mu \mathrm{m}$

Fig. 6 denotes the multi-frequency electrical resistance (i.e. the real part of the measured impedance) of the aluminium films (44 $\mu \mathrm{m}$ and $66 \mu \mathrm{m}$ ) from both transmitter-receiver 1 and transmitter-receiver 2 with the sensor lift-off of $1 \mathrm{~mm}$ and 4 $\mathrm{mm}$. It can be observed that a larger sensor lift-off will result in an attenuation of the sensor response, which is due to the reduced interaction between the sensor and metallic films. Moreover, the multi-frequency resistance curve of transmitterreceiver 2 is slightly left-shifted and larger than that of transmitter-receiver 1 , which is determined by the radius of the coil (as can be seen from equation (9) and (10)). As Fig. 7 depicts, a similar trend can be seen from the multi-frequency resistance curve of the copper films.

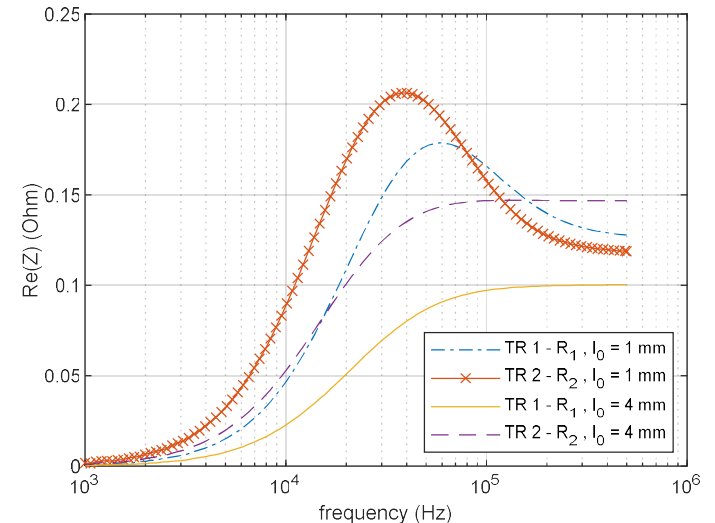

(a)

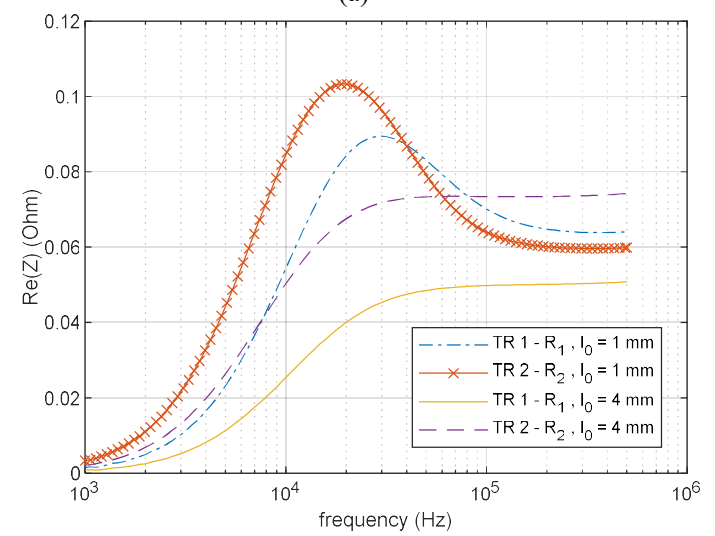

(b)

Fig. 7 Experimental data for the multi-frequency electrical resistance (real part of the measured impedance) of copper films under the sensor lift-off of 1 and 4 $\mathrm{mm}$ (a) $30 \mu \mathrm{m}$ (b) $60 \mu \mathrm{m}$

\section{B. Reconstructed thickness}

Further, the measured electrical resistance of both aluminium and copper films has been inputted in the thickness formulation - equation (30) for the thickness reconstruction. In Fig. 8, the reconstructed thickness of the aluminium film is gradually reduced and close to the actual value under the increased frequency. After a specific frequency, the reconstructed thickness is slightly lower than the actual value but almost immune to the frequency changes. A similar trend can be found in Fig. 9 for the thickness reconstruction of the copper films. It can be observed that the thickness of the films can be reconstructed preciously with only a small error of less than $2 \%$ (with a maximum sensor lift-off of $4 \mathrm{~mm}$ ).

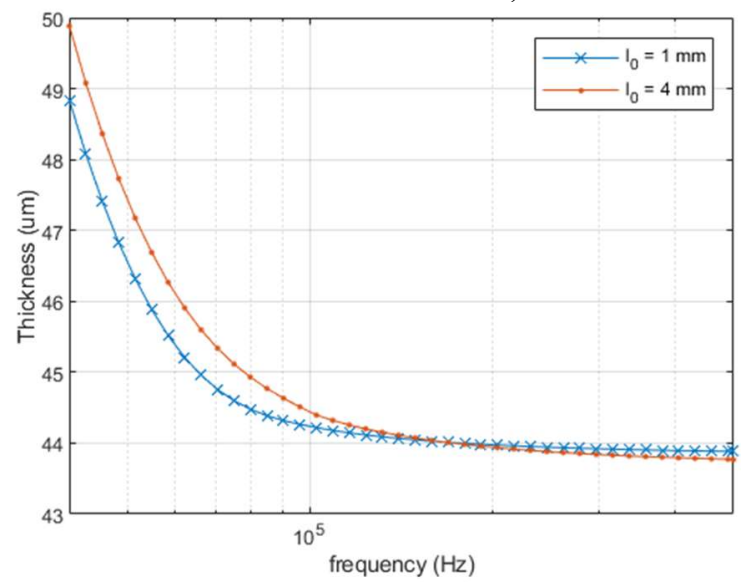

(a) 


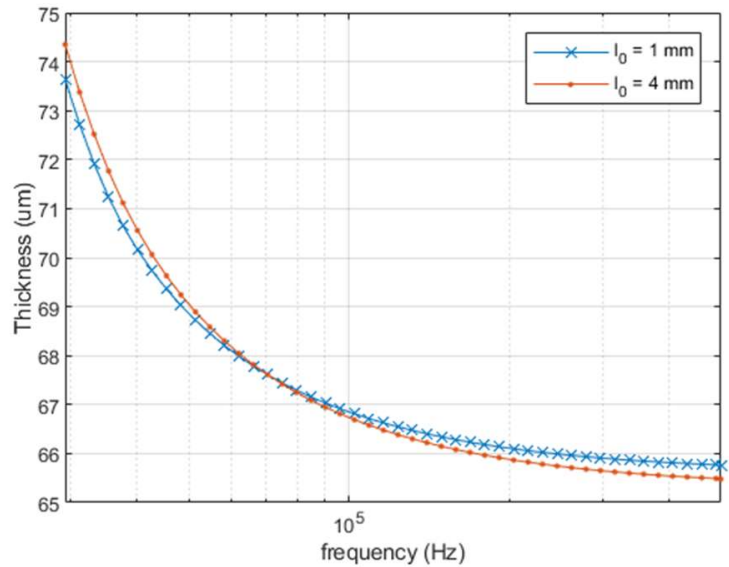

(b)

Fig. 8 Reconstructed thickness of aluminium films under different working frequencies with the sensor lift-off of 1 and $4 \mathrm{~mm}$ (a) $44 \mu \mathrm{m}$ (b) $66 \mu \mathrm{m}$

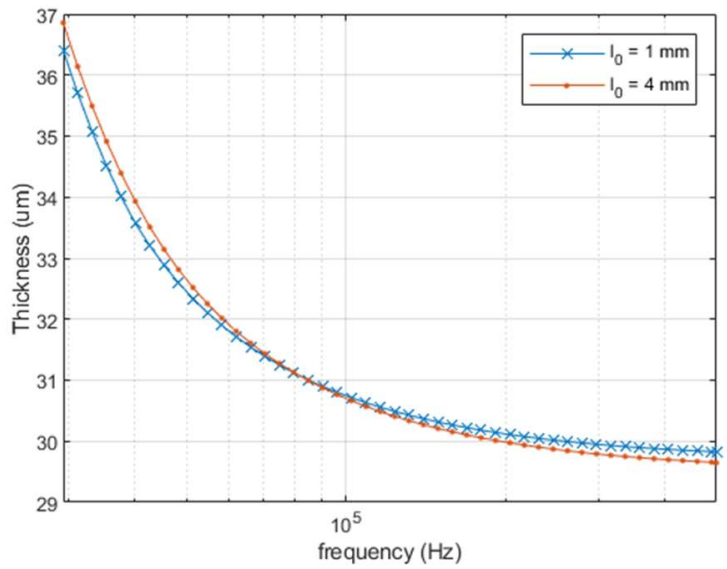

(a)

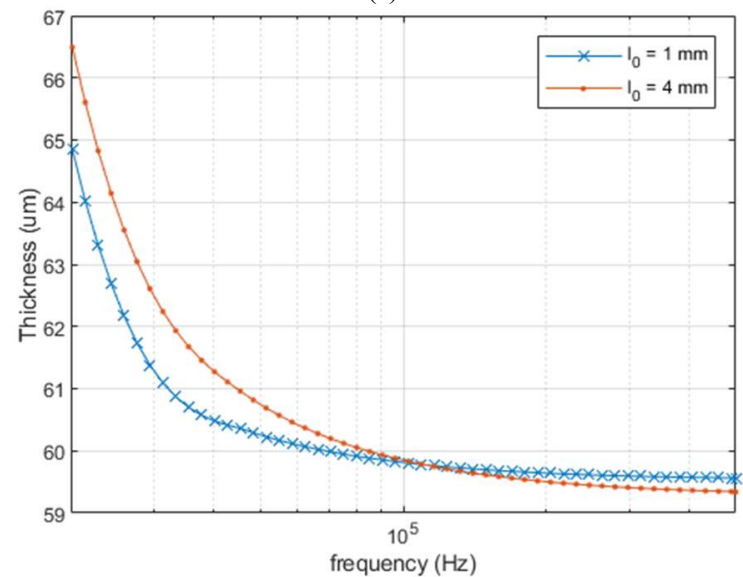

(b)

Fig. 9 Reconstructed thickness of copper films under different working frequencies with the sensor lift-off of 1 and $4 \mathrm{~mm}$ (a) $30 \mu \mathrm{m}$ (b) $60 \mu \mathrm{m}$

\section{Lift-off effects}

Since the reconstructed thickness of the metallic film is more accurate under the high frequency (not higher than the skineffect frequency), the working frequency is selected as $500 \mathrm{kHz}$ for the further accuracy analysis of an extended sensor lift-offs. It can be seen from Fig. 10 and 11 that, the reconstructed thickness gradually drifts away from the actual value but becomes almost immune to the lift-off of the sensor. For a maximum sensor lift-off of $6 \mathrm{~mm}$, the reconstruction error is less than $2 \%$. However, as can be seen from Fig. 12, it has been found further significantly increased sensor lift-offs could result in an inaccurate value when using (30), which is due to the small value of measured electrical resistance. Therefore, the reconstructed thickness scenario can only be valid on a reasonable range of sensor lift-offs (particularly smaller than 12 $\mathrm{mm}$ ). In Fig. 12, another sample - brass film with an electrical conductivity of $15.9 \mathrm{MS} / \mathrm{m}$ and thickness of $100 \mu \mathrm{m}$ has been added for further comparison of the reconstruction on different metals. Due to the limitation of the skin effect (31), the limit of the thickness that can be measured are 116, 92, and $178 \mu \mathrm{m}$ for the aluminium, copper, and brass films.

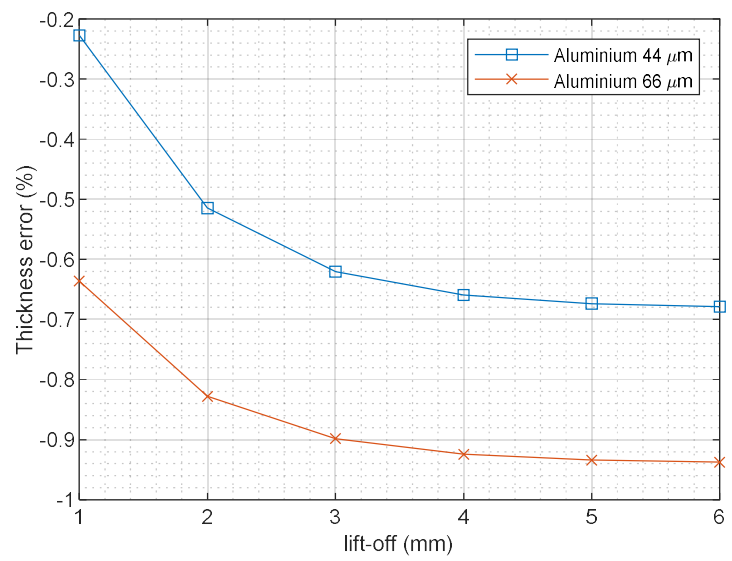

Fig. 10 Error of the reconstructed thickness for aluminium films (44 $\mu \mathrm{m}$ and 66 $\mu \mathrm{m})$ under different lift-offs of the sensor

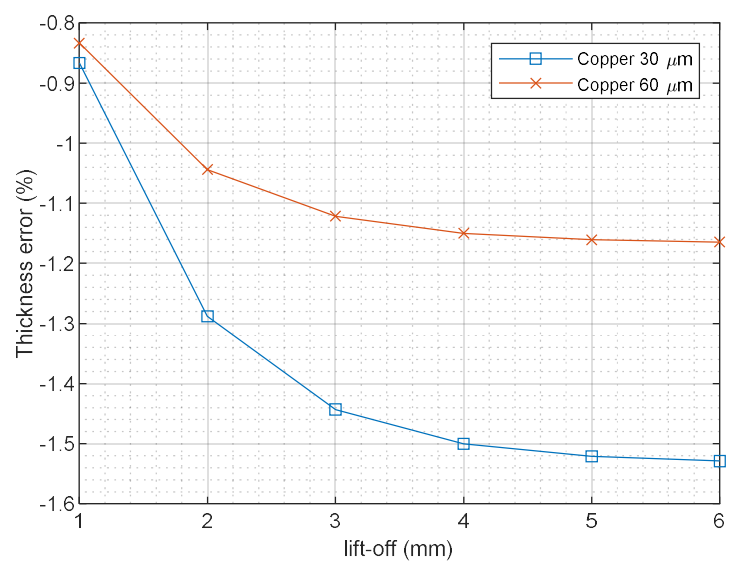

Fig. 11 Error of the reconstructed thickness for copper films ( $30 \mu \mathrm{m}$ and $60 \mu \mathrm{m})$ under different lift-offs of the sensor

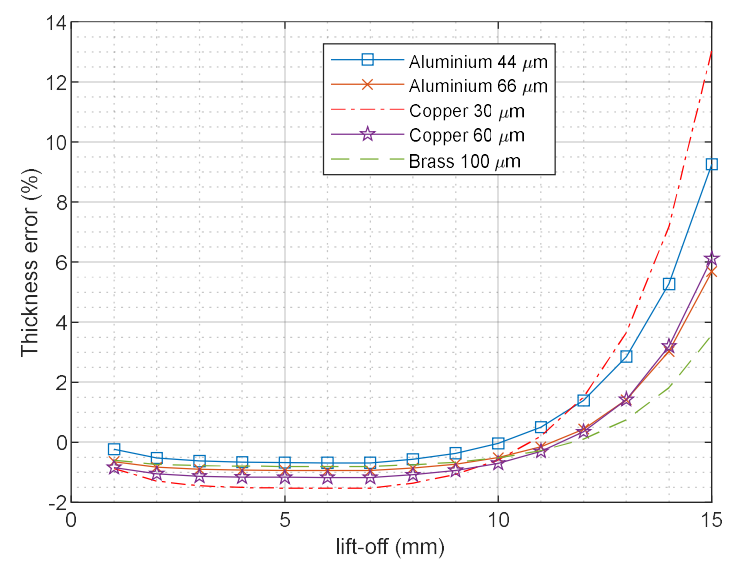

Fig. 12 Error of the reconstructed thickness for different samples with an extended lift-off of the sensor 


\section{CONCLUSIONS}

In this paper, a novel algorithm has been proposed for the thickness reconstruction of non-magnetic metal films. First, an air-core sensor with three circular coils enwound has been designed for the measurement of the electrical resistance from the sensor response. By combining the measurement of two sensing pairs (with one transmitter and two receivers), the thickness of the metallic film (non-magnetic) can be reconstructed. The thickness algorithm has considered an approximation of the Bessel terms, which involves a simple formulation instead of integrating over the whole domain of $\alpha$ when using the Dodd-Deed analytical method. Consequently, the proposed thickness could achieve a fast feedback response, which can therefore potentially be applied for the online realtime thickness monitoring in the future. The thickness reconstruction scenario reaches its highest performance under a high working frequency (best performance is under $500 \mathrm{kHz}$ in this paper). From the experiments on aluminium and copper films with different thicknesses, the proposed algorithm is proved can reconstruct the thickness of the film preciously, with a small error of less than $2 \%$. Moreover, the thickness measurement scheme is valid in a small range of sensor lift-offs, as further increased lift-off will significantly reduce the interaction between the sensor and metal. Therefore, it is worth to investigate an optimal sensor geometry (maybe combine the pancake coil with helix coil) for a large range of lift-offs in the future.

\section{ACKNOWLEDGEMENT}

This work was supported by [UK Engineering and Physical Sciences Research Council (EPSRC)] [grant number: EP/P027237/1] [title: Real-time In-line Microstructural Engineering (RIME)]

\section{REFERENCES}

[1]. J. C. Moulder, E. Uzal, and J. H. Rose, "Thickness and conductivity of metallic layers from eddy current measurements," Review of Scientific Instruments, vol. 63, no. 6, 1992.

[2]. X. Chen and Y. Lei, "Electrical conductivity measurement of ferromagnetic metallic materials using pulsed eddy current method," NDT \& E International, vol. 75, pp. 33-38, 2015.

[3]. G. Yang, G. Dib, L. Udpa, A. Tamburrino and S. S. Udpa, "Rotating Field EC-GMR Sensor for Crack Detection at Fastener Site in Layered Structures," IEEE Sensors Journal, vol. 15, no. 1, pp. 463-470, Jan. 2015.

[4]. W. Li, X. Yuan, G. Chen, J. Ge, X. Yin and K. Li, "High sensitivity rotating alternating current field measurement for arbitrary-angle underwater cracks," NDT\&E International, vol. 79, pp. 123-131, Apr. 2016.

[5]. D. Vasic, V. Bilas and D. Ambrus, "Pulsed eddy-current nondestructive testing of ferromagnetic tubes," IEEE Transactions on Instrumentation and Measurement, vol. 53, no. 4, pp. 1289-1294, Aug. 2004.

[6]. I. Z. Abidin, C. Mandache, G. Y. Tian and M. Morozov, "Pulsed eddy current testing with variable duty cycle on rivet joints," NDT \& E International, vol. 42, no. 7, pp. 599-605, 2009.

[7]. Y. He, G. Tian, H. Zhang, M. Alamin, A. Simm and P. Jackson, "Steel Corrosion Characterization Using Pulsed Eddy Current Systems," IEEE Sensors Journal, vol. 12, no. 6, pp. 2113-2120, June 2012.

[8]. M. Lu, et al. "Acceleration of frequency sweeping in eddy-current computation," IEEE Transactions on Magnetics, vol. 53, pp. 1-8, 2017.

[9]. Y. Shin, D. Choi, Y. Kim and S. Lee, "Signal characteristics of differential-pulsed eddy current sensors in the evaluation of plate thickness," NDT \& E International, vol. 42, no. 3, pp. 215-221, 2009.
[10]. D. Wen, M. Fan, B. Cao, B. Ye and G. Tian, "Extraction of LOI Features from Spectral Pulsed Eddy Current Signals for Evaluation of Ferromagnetic Samples," IEEE Sensors Journal, vol. 19, no. 1, pp. 189195, 1 Jan.1, 2019

[11]. G. Y. Tian and A. Sophian, "Reduction of lift-off effects for pulsed eddy current NDT," NDT \& E International, vol. 38, no. 4, pp. 319-324, 2005.

[12]. A. V. Egorov, V. V. Polyakov, D. S. Salita, E. A. Kolubaev, S. G. Psakhie, A. G. Chernyavskii, I. V. Vorobei, "Inspection of aluminum alloys by a multi-frequency eddy current method," Defence Technology, vol. 11, no. 2, pp. 99-103, 2015.

[13]. W. Yin and A.J. Peyton, "Thickness measurement of non-magnetic plates using multi-frequency eddy current sensors," NDT \& E International, vol. 40, no. 1, pp. 43-48, 2007.

[14]. W. Zhou, M. Lu et al. "Three-dimensional electromagnetic mixing models for dual-phase steel microstructures," Applied Sciences, vol. 8, pp. 529, 2018.

[15]. T. Theodoulidis and E. E. Kriezis, "Eddy current canonical problems (with applications to nondestructive evaluation)", Material Science, 2006.

[16]. G. Tytko and L. Dziczkowski, "E-Cored Coil With a Circular Air Gap Inside the Core Column Used in Eddy Current Testing," IEEE Transactions on Magnetics, vol. 51, no. 9, pp. 1-4, Sept. 2015.

[17]. W. Yin, A. Peyton and S. J. Dickinson, "Simultaneous Measurement of Distance and Thickness of a Thin Metal Plate With an Electromagnetic Sensor Using a Simplified Model," IEEE Transactions on Instrumentation and Measurement, vol. 53, no. 4, pp. 1135-1138, 2004.

[18]. M. Lu, H. Xu, W. Zhu, L. Yin et al. "Conductivity Lift-off Invariance and measurement of permeability for ferrite metallic plates," NDT \& E International, vol. 95, pp. 36-44, Apr. 2018.

[19]. M. Lu, R. Huang, W. Yin, Q. Zhao, and A. Peyton, "Measurement of permeability for ferrous metallic plates using a novel lift-off compensation technique on phase signature, " IEEE Sensors Journal, vol. 19, no. 17, pp. 7440-7446, 1 Sept.1, 2019.

[20]. M. Lu, et al. "Determination of the magnetic permeability, electrical conductivity, and thickness of ferrite metallic plates using a multifrequency electromagnetic sensing system," IEEE Transactions on Industrial Informatics, vol. 15, pp. 4111-4119, 2019.

[21]. I. N. Prassianakis and N. I. Prassianakis, "Ultrasonic testing of nonmetallic materials: concrete and marble," Theoretical and Applied Fracture Mechanics, vol. 42, no. 2, pp. 191-198, Nov. 2004.

[22]. J. Kral, R. Smid, H. M. G. Ramos and A. L. Ribeiro, "The lift-off effect in eddy currents on thickness modeling and measurement," IEEE Transactions on Instrumentation and Measurement, vol. 62, no. 7, pp. 2043-2049, 2013.

[23]. A. L. Ribeiro, H. G. Ramos and J. C. Arez, "Liftoff insensitive thickness measurement of aluminum plates using harmonic eddy current excitation and a GMR senso," Measurement, vol. 45, no. 9, pp. 2246-2253, 2012.

[24]. D. F. He and M Yoshizawa, "Dual-frequency eddy-current NDE based on high-Tc rf SQUID," Physica C: Superconductivity, vol. 383, no. 3, pp. 223-226, 2002.

[25]. G. Y. Tian, and A. Sophian, "Reduction of lift-off effects for pulsed eddy current NDT,". NDT \& E International, vol. 38, no. 4, pp. 319-324, 2005.

[26]. C. C. Tai, J. H. Rose, and J. C. Moulder, "Thickness and conductivity of metallic layers from pulsed eddy-current measurements," Review of Scientific Instruments, vol. 67, no. 11, Aug. 1996.

[27]. E. Pinotti and E. Puppin, "Simple Lock-In Technique for Thickness Measurement of Metallic Plates," IEEE Transactions on Instrumentation and Measurement, vol. 63, no. 2, pp. 479-484, Feb. 2014.

[28]. J.R.S. Avila, M. Lu et al. "Accurate measurements of plate thickness with variable lift-off using a combined inductive and capacitive sensor," NDT \& E International, vol. 110, pp. 102202, 2020.

[29]. M. Lu, L. Yin, A. J. Peyton and W. Yin, "A novel compensation algorithm for thickness measurement immune to lift-off variations using eddy current method," IEEE Transactions on Instrumentation and Measurement, vol. 65, no. 12, pp. 2773-2779, Dec. 2016.

[30]. M. Lu, X. Meng, W. Yin, Z. Qu, F. Wu, J. Tang, et al., “Thickness measurement of non-magnetic steel plates using a novel planar triple-coil sensor," NDT \& E International, vol. 107, 2019. 
[31]. R. Huang, M. Lu, A. Peyton, and W. Yin, "Thickness measurement of metallic plates with finite planar dimension using eddy current method," IEEE Transactions on Instrumentation and Measurement, vol. 69, no. 10, 2020. Doi:10.1109/TIM.2020.2987413.

[32]. M. Lu et al. "Measurement of ferromagnetic slabs permeability based on a novel planar triple-coil sensor," IEEE Sensors J., vol. 20, no. 6, pp. 2904-2910, 2020.

[33]. M. Lu, W. Zhu, L. Yin, A. J. Peyton, W. Yin, and Z. Qu, "Reducing the lift-off effect on permeability measurement for magnetic plates from multifrequency induction data," IEEE Transactions on Instrumentation and Measurement, vol. 67, no. 1, pp. 167-174, Jan. 2018.

[34]. C. V. Dodd, and W. E. Deeds, "Analytical solutions to eddy-current probe-coil problems," Journal of applied physics, vol. 39, no. 6, pp. 2829-2838, 1968.

[35]. R. Huang, M. Lu et al. "A novel perturbed matrix inversion based method for the acceleration of finite element analysis in crack-scanning eddy current NDT," IEEE Access, vol. 8, pp. 12438-12444, 2020.

[36]. W. Yin, M. Lu et al. "Custom edge-element FEM solver and its application to eddy-current simulation of realistic 2M-element human brain phantom," Bioelectromagnetics, vol. 39, pp. 604-616, 2018.

[37]. W. Yin, M. Lu et al. "Acceleration of eddy current computation for scanning probes," Insight-Non-Destructive Testing and Condition Monitoring, vol. 60, pp. 547-555, 2018.

[38]. J. Tang et al. "A Novel Efficient FEM Thin Shell Model for BioImpedance Analysis," Biosensors, vol. 10, pp. 69, 2020.

[39]. Y. Xie, M. Lu et al. "Novel Wearable Sensors for Biomechanical Movement Monitoring Based on Electromagnetic Sensing Techniques,” IEEE Sensors J., vol. 20, no. 2, pp. 1019-1027, 2019.

[40]. M. Lu et al. "Forward solver for deep earth exploration and induction logging using custom built Edge-Element FEM technique," Acta Geologica Sinica - English Edition, vol. 93, pp. 302-304, 2019.

[41]. T. Yang, et al. "Level measurement for saline with a small surface area using high frequency electromagnetic sensing technique," Measurement, vol. 101, pp. 118-125, 2017.

[42]. W. Zhou, M. Lu et al. "Three-dimensional electromagnetic mixing models for dual-phase steel microstructures," Applied Sciences, vol. 8, pp. 529, 2018.

[43]. M. Lu et al. "Prediction of the asymptotical magnetic polarization tensors for cylindrical samples using the boundary element method," 2015 IEEE Sensors Applications Symposium (SAS), pp. 1-4, 2015.

[44]. W. Yin et al. "An equivalent-effect phenomenon in eddy current nondestructive testing of thin structures," IEEE ACCESS, vol. 7, pp. 70296 $-70307,2019$

[45]. H. Xu, M. Lu et al. "Imaging Weld Cross-Section Using a Novel Frequency Feature in Multi-Frequency Eddy Current Testing," InsightNon-Destructive Testing and Condition Monitoring, vol. 61, pp. 738743, 2019.

[46]. W. Yin et al. "Permeability invariance phenomenon and measurement of electrical conductivity for ferrite metallic plates," Insight-NonDestructive Testing and Condition Monitoring, vol. 61, pp. 472-479, 2019.

[47]. L. Yin et al. "Detection of corrosion pits based on an analytically optimised eddy current sensor," Insight-Non-Destructive Testing and Condition Monitoring, vol. 60, pp. 561-567, 2018.

[48]. M. Lu et al. "Determining the magnetic permeability of ferrite steel strip by a custom inversion method," Proc. 12th ECNDT, pp. 1-8, 2018.

[49]. J. Tang et al. "Bio-impedance spectroscopy for frozen-thaw of biosamples: Non-contact inductive measurement and finite element (FE) based cell modelling," Journal of Food Engineering, vol. 272, pp. 109784, 2020. 